\title{
Influence of Obstacles on the Use of the Danger Zone on Railway Platforms \\ Jasmin Thurau ${ }^{1}$, Nicolas Keusen ${ }^{2}$ \\ ${ }^{1}$ SBB AG, Technologic Asset Management \\ Hilfikerstrasse 3, 3000 Bern 65, Switzerland \\ jasmin.thurau@sbb.ch \\ ${ }^{2} \mathrm{BAV}$, Dept. of Security \\ Ittigen, Switzerland \\ Nicolas.Keusen@bav.admin.ch
}

\begin{abstract}
Growing passenger numbers and the lack of space available led to research on pedestrians' behaviour on railway platforms in Switzerland. By using stereo sensors, pedestrians' tracks were collected on a platform in the train station of Bern. The analysis of pedestrians stepping into the danger zone showed clearly that obstacles have a large influence on the frequencies of pedestrians using the danger zone. By presenting four hypotheses the effect of obstacles on pedestrians' use of the danger zone on train station platforms is investigated.
\end{abstract}

Keywords: railway platforms, pedestrian behaviour, use of danger zone, influence of obstacles

\section{Introduction}

Passenger numbers grew significantly within the last years and are expected to increase further in the future. This trend results in more crowded platforms and, especially during peak hours, the capacity of platforms is nearly reached.

Latest research analysed the relation between pedestrian density on platforms and the number of pedestrians entering the danger zone [1]. Thurau et al. (2017) show that there is still further research needed when defining the maximum number of passengers allowed on platforms. By using highly accurate sensor data [2], this paper focuses on where pedestrians on railway platforms tend to use the danger zone more often.

For this paper the Swiss definition of the danger zone is used (Fig. 1). It is defined as follows: The danger zone is the area next to the tracks, which shouldn't be used by passengers when no train occupies or a train drives through the station. The safety zone is the area which shall be used by passengers for movements and waiting. The danger zone starts with the safety line, which itself is still within the safety zone.

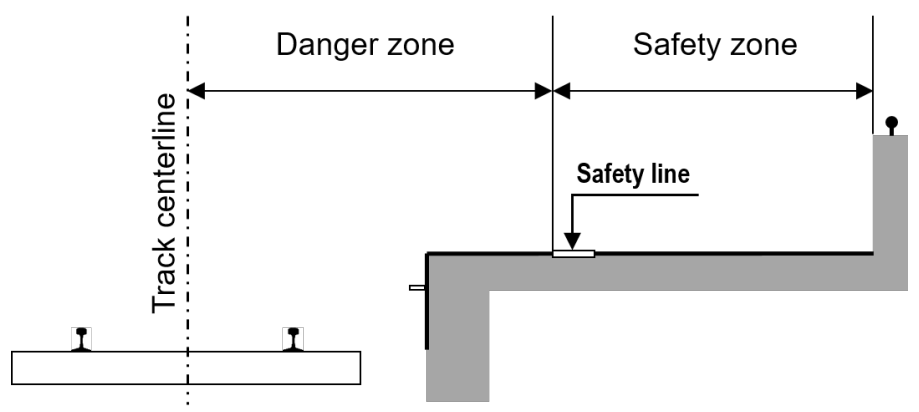

Fig. 1 Definition of the danger zone

\section{Hypotheses}

In order to analyse the influence of obstacles on railway platforms on the use of the danger zone, the following hypotheses have been presented: 
H1: Next to obstacles the number of people stepping into the danger zone is significantly higher than in areas without any obstacles.

H2: The share of people using the danger zone rises when the safety zone gets smaller.

H3: The duration of people using the danger zone is significantly higher next to obstacles than in areas without any obstacles nearby.

H4: The average walking speed of pedestrians using the danger zone decreases with an increase of time people use the danger zone.

$\mathrm{H} 1$ and $\mathrm{H} 2$ are derived from the idea that less space for overtaking, due to obstacles or other pedestrians, leads to a higher number of pedestrians stepping into the danger zone [3].

Under the assumption that safety zones are similarly crowded, H3 is based on the thought that overtaking pedestrians next to larger obstacles require longer distances within the danger zone, in comparison to short obstacles [3].

$\mathrm{H} 4$ results from the idea that pedestrians use the danger zone for the shortest possible time period and therefore speed up for overtaking. Pedestrians that do not realize the potential threat when being within the danger zone do not change their walking speed, as they do not make a difference between both platform zones.

\section{Analysis}

\subsection{Data collection and data set}

In order to investigate the hypotheses, tracking data of pedestrians on a railway platform in the train station of Bern, Switzerland is used. The data is collected with high precision sensors [2]. The data was aggregated to $\mathrm{x}$ - and $\mathrm{y}$-coordinates per person per second. Basic characteristics per pedestrian, such as walking speed, were calculated. By combining this data with information describing the presence or absence of trains on the tracks, it was possible to select only the situations when pedestrians were using the danger zone without a train occupying the track. These situations are considered as potentially unsafe and are therefore relevant for the investigation of the hypotheses. The analysis is based on data for weekdays and day time (6am to 8pm) for the month of September 2016 ( 21 weekdays). The number of trains is 2 to 4 per hour and track.

Fig. 2 shows the sensor equipped area of platform 3/4 in Bern. It consists of a shorter obstacle (staircase) to the left (zone 1), a longer obstacle (ramp) in the middle (zone 2) and small obstacles to the right (zone 5). Zone 3 represents the zone were passengers enter or leave the platform by using the ramp; zone 4 has no obstacles. In the following the platform is going to be separated in the middle, parallel to the tracks, so that zone 1 at track 3 is named 1.3, zone 2 at track 4 is named 2.4 and so on.

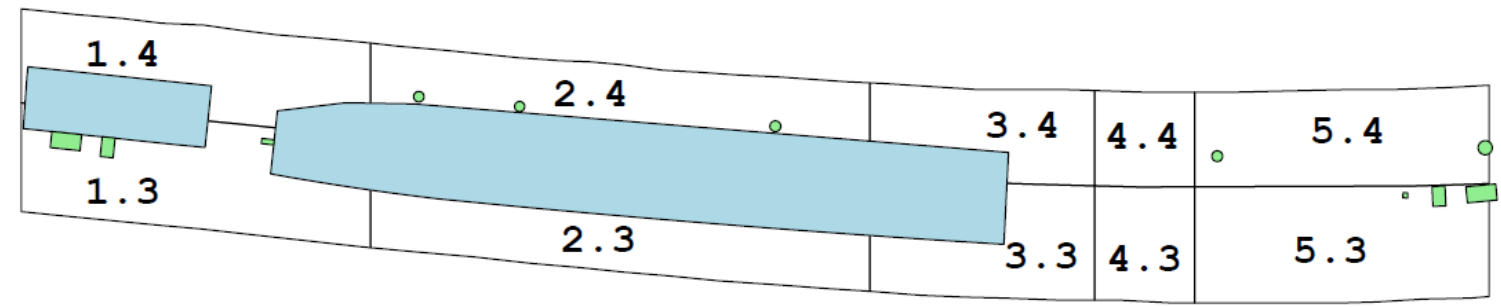

Fig. 2 Platform layout Bern - tracks 3 and 4

For the analysis of hypothesis 1,2 and 4 the platform is separated into zones of $1 \mathrm{~m}$ length. This results into zones from 1.3 until 60.3 at track 3 and 1.4 to 60.4 at track 4 , as 60 meters length of the platform is equipped with sensors.

\subsection{Results for H1 \& H2: number of uses and share of people using the danger zone}

$\mathrm{H} 1$ and $\mathrm{H} 2$ can't be rejected: The analysis shows a higher number of uses of the danger zone next to obstacles. Overall results for both tracks show a median number of uses of the danger zone during 
daytime at weekdays of 7.19 uses per hour in zones with less than $2 \mathrm{~m}$ safety zone. For safety zones wider than $2 \mathrm{~m}$ the median is 3.65 , which is significantly lower. Table 1 shows detailed results including upper and lower quartile. The median share of people using the danger zone next to obstacles and with smaller safety zones $(11 \%)$ is significantly higher than the share of people using the danger zone at wider safety zones $(3 \%)$. The lower quartile of small safety zones $(9 \%)$ is higher than the upper quartile of wider safety zones $(4 \%)$.

Table 1 number and share of uses of the danger zone with different width of safety zone

\begin{tabular}{|l|r|r|}
\hline & \multicolumn{3}{|l|}{$\begin{array}{l}\text { Uses of the danger } \\
\text { zone }\end{array}$} \\
\hline width of safety zone & $<\mathbf{2 m}$ & $\mathbf{2} \mathbf{2 m}$ \\
\hline upper quartile & 9.5 & 5.5 \\
\hline median & 7.19 & 3.65 \\
\hline lower quartile & 5.76 & 2.58 \\
\hline
\end{tabular}

\begin{tabular}{|l|r|r|}
\hline & \multicolumn{2}{|l|}{$\begin{array}{l}\text { share of people using } \\
\text { the danger zone }\end{array}$} \\
\hline width of safety zone & $<\mathbf{2 m}$ & $\mathbf{2 m}$ \\
\hline upper quartile & $15 \%$ & $4 \%$ \\
\hline median & $11 \%$ & $3 \%$ \\
\hline lower quartile & $9 \%$ & $2 \%$ \\
\hline
\end{tabular}

\subsection{Results for H3: duration of use of the danger zone}

H3 can't be rejected: The highest median duration of people's use of the danger zone was measured in zone 2.3, this is the zone next to the ramp, representing the smallest safety zone on the platform. The results for zone 2.4 are the second highest, although the safety zone is slightly smaller (see Table 2). The reason might be the lower frequency of use of this side of the ramp. This shows that additional factors, such as person density, influence pedestrians use of the danger zone (see also [1]).

Table 2 duration of use of danger zone with different width of safety zone [s]

\begin{tabular}{|c|c|c|c|c|c|c|c|c|c|c|c|c|}
\hline \multirow[b]{2}{*}{ zone } & \multicolumn{12}{|c|}{ average duration in danger zone } \\
\hline & 1.3 & 1.4 & 2.3 & 2.4 & \begin{tabular}{|r|}
3.3 \\
\end{tabular} & \begin{tabular}{|r|}
3.4 \\
\end{tabular} & 4.3 & 4.4 & 5.3 & 5.4 & & \\
\hline $\begin{array}{l}\text { average width of } \\
\text { safety zone }\end{array}$ & $2.68 \mathrm{~m}$ & $2.22 \mathrm{~m}$ & $1.73 m$ & $1.72 \mathrm{~m}$ & $2.67 \mathrm{~m}$ & $2.44 \mathrm{~m}$ & $4.10 \mathrm{~m}$ & $3.30 \mathrm{~m}$ & $4.04 \mathrm{~m}$ & $3.33 \mathrm{~m}$ & $<2 m$ & $>2 \mathrm{~m}$ \\
\hline upper quartile & 2.13 & 0.86 & 20.14 & 6.10 & 6.72 & 1.33 & 9.69 & 4.50 & 3.45 & 1.02 & 12.55 & 3.16 \\
\hline median & 1.41 & 0.46 & 14.53 & 4.53 & 3.50 & 0.67 & 4.00 & 3.25 & 2.27 & 0.59 & 9.79 & 2.27 \\
\hline lower quartile & 0.86 & 0.25 & 10.66 & 3.06 & 1.72 & 0.33 & 1.81 & 2.00 & 1.31 & 0.34 & 7.74 & 1.59 \\
\hline
\end{tabular}

\subsection{Results for H4: walking speed and duration in danger zone}

Data reliability is not high enough to calculate the duration within the danger zone per person. The overall time of any person in the danger zone can be computed, but not the duration of use of the danger zone for a certain ID. Therefore, speed and distance of a certain ID can't be compared, but analysis of H3 was possible.

\section{Conclusion}

It is shown that the available width of the safety zone clearly influences number, share and duration of use of the danger zone by pedestrians. It can be found that at around two meters safety zone we can find a different behaviour of pedestrians regarding the use of the danger zone. This parameter needs further analysis, especially at different train stations. In the next step, a model including the width of the safety zone and the person density shall be developed.

\section{References}

[1] J. Thurau, J. van den Heuvel, N. Keusen, M. van Ofwegen und S. Hoogendoorn, „Influence of Pedestrian Density on the Use of the Danger Zone at Platforms of Train Stations", Bern / Utrecht, 2017.

[2] J. van den Heuvel, J. Thurau, M. Mendelin, R. Schakenbos, M. van Ofwegen und S. Hoogendoorn, „An application of new pedestrian tracking sensors for evaluating platform safety risk at Swiss and Dutch train stations“, Utrecht / Bern, 2017.

[3] N. Keusen et al., „Safety Distances on Platforms”, Bern, 2011. 